\title{
COMMODITY INDICES RISK AND RETURN ANALYSIS AGAINST LIBOR BENCHMARK
}

\author{
Subhakara Valluri \\ Institute of Economics of the Polish Academy of Sciences ${ }^{1}$, Poland \\ subhakara.valluri@gmail.com
}

\begin{abstract}
This study analyze the risk and return characteristics of commodity index investments against the LIBOR benchmark. Commodity-based asset allocation strategies can be optimized by benchmarking the risk and return characteristics of commodity indices with LIBOR index rate. In this study, we have considered agriculture, energy, and precious metals commodity indices and LIBOR index to determine the risk and return characteristics using estimation techniques in terms of expected return, standard deviation, and geometric mean. We analyzed the publicly available daily market data from 10/9/2001 to 12/30/2016 for benchmarking commodity indices against LIBOR. S\&P GSCI Agriculture Index (SGK), S\&P GSCI Energy Index (SGJ), and S\&P GSCI Precious Metals Index (SGP) are taken to represent each category of widely traded commodities in the regression analysis. Our study uses time series data based on daily prices. Alternative forecasting methodologies for time series analysis are used to cross-check the results. The forecasting techniques used are Holt-Winters Exponential Smoothing and ARIMA. This methodology predicts forecasts using smoothening parameters. The empirical research has shown that the risk of each of the commodity index that represents agriculture, energy, and precious metals sector is smaller compared to its return, whereas LIBOR based interest rate benchmark shows higher risk compared to its return in recession, non-recession and overall periods.
\end{abstract}

Keywords: Standard \& Poor's Goldman Sachs Commodity Index, Holt-Winters Exponential Smoothing, ARIMA, LIBOR. (JEL Classification: C43, G13, G15)

\section{INTRODUCTION}

The financial market in the economy is the most important segment of an economy in terms of measuring economic growth. Investors and borrowers are participants in financial markets. Financial markets are classified into Money Markets and Capital Markets. The money market is for those investors who invest in assets for short-term and borrowers who borrow assets for short-term. Capital markets are for investors and borrowers who are large organizations or entities trading for the long term. Financial markets are driven by investors, financial institutions, banks and business entities. Economic factors or macroeconomic variables are directly or indirectly

1 This research is part of doctoral studies at Institute of Econoics of the Polish Academy of Sciences. correlated with the financial market state. Any small or visible change in the economy has an impact on financial markets. Demand and supply of money in capital markets plays a significant role in determining asset prices. Investors and other market participants enter into a trade or transact based on the current market prices and how much return will the asset give in future on investment. Financial markets fundamental factors take part in decision making of asset class selection for investors. Financial market participants study and analyze various factors for their investment decisions.

A financial derivative is a contract of an underlying asset between buyer and seller with an agreed upon price at a future date. The value of an underlying asset is derived based on market conditions and other economic factors. Some examples of underlying assets are interest rates, commodities, currencies, indices, and stocks. The commodity derivatives 
market is driven by commodity producers, financial entities and investors who want to hedge their assets against future changing prices.

Futures and Options market players are usually hedgers or speculators trading in derivative contracts of underlying such as currency, interest rates, commodities, etc. Hedgers mostly enter into a contract to reduce the risk that may arise out of future price fluctuations. They use specific derivative structured products to reduce or eliminate future price risk. Let us consider a scenario for hedgers in the futures market.

Speculators are the market participants who aim to maximize their profit on investment. They are risk takers and play in futures market purely to make a profit. Speculators use technical analysis and fundamental analysis techniques to forecast future trends and make investment decisions. They also run the risk of losses if their investment turns out to be the other way.

Inflation plays a key role in the determination of market prices. Previous studies have shown that investors may gain from stock and bonds when the market predicts expected inflation. There can be other scenarios where unexpected inflation occurs, and this may result in a cause of concern for stock and equity investors.

Rise in inflation rate causes higher interest rates operational cost increases in terms of raw materials and logistics for manufacturers and large business entities. Demand for loans in such situations goes more elevated than usual. Banks and financial institutions take advantage of increased demand for borrowings. They raise interest rates to make money out of amounts they have by lending funds on higher interest rates. The cycle of demand and supply of resources becomes responsible for the economic shift. Changes in interest rates also affect different types of investments. Stock companies make lower profits to pay high interest rates, and stock prices may fall due to rising interest rates. Bond markets prices are determined based on the number of buy and sell transactions. Increase in interest rate causes a fall in bond prices and vice versa. Commodity prices and interest rates also have shown linkages in history. Agriculture, energy and precious metals prices go up when there is a fall in interest rates and vice versa. Frankel (2012) highlighted in his studies that interest rate movement is a prime factor while forecasting commodity futures prices. He also pointed out that characteristics of commodities are important to be considered while assessing movements of interest rates in determining futures prices.

In recent times, passive investments via indices of underlying assets have shown visibility. Investors take diversification decisions by investing in indices. Change in price movements of index funds is related to market factors. Instead of investors tracking the market movements, price of indices will tell you the direction of where the market is going. There are different approaches to index investments. An investor can decide whether he wants to invest in an index that captures the entire stock market or in the index that covers sub-market-sectors, for example - stocks of the small, medium, large companies. These are some advantages of index investments. In this study, we are taking commodity indices and interest rate benchmark index to evaluate their risk and return characteristics.

Commodity indices are the benchmark to measure the performance of underlying commodity prices over a period. Each index tracks the performance of the commodity involved in that index. Most commodity indices are traded in futures markets via exchanges. The commodity indices are indirect access of commodities to investors trading in the market without entering into commodity futures markets. Indices also act as a source of information and performance benchmarks to forecast trends in cash and futures segments. Commodity indices also help investors or their fund managers in deciding asset allocation strategies. There are several economic factors such as increasing demand for commodities from developing countries (China, India, etc.), increase in interest rates, strong monetary policy, and increasing demand for energy that would impact commodity prices in future. Investors can take advantage of these factors by investing in commodities as part of their diversification approach.

Interest rate index is referred to as the benchmark rate of interest on the computation of payment schedules and amortization schedules of financial products such as mortgage or loans. Market participants or investors choose financial products based on the bank rates and banks determine the interest rate using the standard index rate. They use index rate as input to determine the interest rate of their financial products. Based on the index rate, they can estimate the future interest rate movements. Interest rate index with different maturity dates is used in different short-term and long-term financial products.

Popular interest rate indices are London Interbank Offered Rate (LIBOR), Treasuries Constant Maturities Index, and Federal Funds Reserve Rate, etc. Banks in London uses LIBOR as the interest rate at which they are willing to lend money to each other in money markets. Federal Funds Reserve rates are used by the banks that are creditworthy and lend overnight funds to each other. National Average Contract Mortgage Rate (NACR) is an index rate used in housing loans used by lenders. This rate is published monthly and very low volatile.

The primary objective of our study is to highlight return and risk characteristics of commodity index investment against risk and return of LIBOR index rate that may further help investors to get insights on their investments. Asset allocation is another aspect that investors may look at by benchmarking the risk and return characteristics of commodity indices with LIBOR. This study may further open the doors for analyzing the returns of these two financial products under different economic conditions.

In this study, we have considered agriculture, energy, and precious metals commodity indices and LIBOR index to determine the return characteristics and compare their returns using estimation techniques in terms of expected return, standard deviation, and geometric mean. To give the study more accuracy, alternative forecasting methodologies for time series analysis are used. The forecasting techniques used are Holt-Winters Exponential Smoothing and ARIMA. The methodology predicts forecasts using smoothening parameters as discussed in the Methodology section. 


\section{LITERATURE REVIEW}

Scherr and Madsen (1983) conducted an observational study on determining the relationship between real interest rates and agricultural commodity prices. They highlighted that the higher interest rates in 1978 showed some behavior in determining agricultural commodity prices. Their observations were based on unusual higher rates of interest, lower rates at the time of inflation and reducing rates for domestic consumption. They also covered the impact of agriculture commodity prices in near-by future.

Gruber and Vigfusson (प2018) examined the effect of interest rates in the volatile market and its relationship with commodity prices. Their study observed that lower interest rates would make the commodity market less volatile and would lead to higher commodity prices assuming shocks are persistent. They showed an inverse relationship between the interest rate and correlation for metal prices. Their research suggested distinguishing financial implications and fundamental factor while measuring commodity price correlation.

Reicher and Utlaut (2010) conducted studies on determining the relationship between oil prices and nominal interest rates using VAR analysis. They discovered a strong positive correlation between oil prices and long-run interest rates, stability in interest rates and short-run oil prices, no correlation between oil prices and productivity and no change in correlation between oil prices and unemployment. The study concluded that the country's monetary policy is a major factor impacting oil prices in the long run.

Nordin et al. (2014) examined the impact of palm oil, oil prices and gold prices, interest rate and exchange rate on the performance of Malaysian stock market returns. He had taken the bounds test approach and the results of the study showed the strong impact of palm oil prices, interest rate and exchange rate on the stock market index returns, no impact of gold prices and oil prices on stock market index returns. He conducted co-integration analysis taking multiple variables identifying the impact on Malaysian stock market index returns.

Sari and Soytas (2006) investigated the relationship between oil price changes and macroeconomic variables such as stock returns and interest rates. The study results indicated that oil prices were unaffected the stock returns in Turkey. There was no significant evidence that showed a direct relationship between macroeconomic variables and changes in oil prices.

Akram (2009) conducted a study to analyze the factors such as exchange rate and real interest rates affecting commodity price fluctuations. The analysis was conducted using a structural VAR model. The study results found that there was a significant increase in commodity prices in response to a decrease in real interest rates. Changes in interest rates showed movements in oil prices and raw material prices. The same was depicted with the exchange rate. Weaker dollar rate leads to an increase in commodity prices. Both variables interest rate and exchange rate found substantial in commodity price fluctuations.

Schnabel (2010) performed a study to examine linkages between changes in interest rates and commodity spot prices.
He used the cost-of-carry model taking the commodity spot and futures prices to measure the effect of changes in interest rates. The results indicated that an increase in interest rate would decrease the spot price. This result was found under mean-reverting expectations. Under the test of invariant expectation, no linkage found between interest rate change and the spot prices. Momentum expectation test showed causality between interest rates and spot prices. Under this test, it was found that an increase in the interest rates caused a rise in spot prices and vice versa.

Kohlscheen et al. (2016) analyzed the relationship between exchange rates and commodity prices. He performed various statistic tests in predictive analysis and found that commodity prices and exchange rates were highly correlated economically and statistically. The commodity price-exchange rate linkage remained unaffected under changes in uncertainty and global risks. The study provided a base to further research on finding to what extent the economic factors are responsible for commodity price developments.

Günay (2015) examined the correlation between liquidity with the overnight $(\mathrm{ON})$ LIBOR rates and stock market price movements. He had taken the scenario of the mortgage crisis of 2008 and considered the countries such as Portugal, Italy, Ireland, Greece, Spain, and Turkey. The empirical analysis was conducted using Fully Modified OLS, Canonical Cointegrating Regression, and Dynamics Least Squares tests. These tests determined the direction of the relationship between stock market price movements and LIBOR rate movements. The increase in ON LIBOR rates indicated a decrease in Turkish and Spanish market liquidity.

Tafa (2015) explained how exchange rate fluctuations impact on interest rate movements. He conducted empirical analysis using regressions to examine the relationship among exchange rate and interests in Albania. The test results showed an increase in interest rates influenced exchange rates positively. Apart from interest rates, other variables such as income level, inflation, government policies and speculation on FX rates also affected exchange rate fluctuations.

Foerster and Sapp (2003) addressed Canadian stock prices and interest rates in his research and performed analysis to find a correlation between prevailing interest rates and stock prices. He found results were different in expansion and recession time periods. He also found that interest rate was highly negatively correlated with returns in industries such as infrastructure and less negatively correlated with returns for consumer product industries. In addition to that, the results also showed positive linkages between returns of resource-based industries and interest rate change. Various observations were found in this regression analysis.

Covrig et al. (2004) conducted studies on TIBOR/LIBOR and the determinants of the 'Japan Premium'. The study indicated that the changing TIBOR-LIBOR spread affects credit risk associated with Japan premium. The spread is a model parameter of this study. Interest rate and stock price effects have an influence on the variance of spread.

Moss and Moss (12010) examined the relationship between bank common stock index price and the interest rate on 
Treasury securities. He also analyzed the correlation between interest rate term structure and bank stock prices. Multiple linear regressions were used to examine variables affecting bank stock prices. The results stated that bank stock prices were affected by changes in interest rates. He mentioned that this study would be helpful for decision making of including bank stocks in investors and bank managers portfolio.

Braml (12016) conducted studies to investigate the integrity of LIBOR as trillions of US dollar products are associated with it. The studies took interest rate parity approach to find out the behavior of LIBOR at the macro and micro level. The macro level analysis showed significant deviation in LIBOR compared to other short-term interest rates. Microlevel analysis indicated there were significant effects on the LIBOR fixing process due to potential manipulation of rates. Irregular behavior was detected if there were manipulation of LIBOR in the rate-setting process.

All the studies above are conducted on various factors affecting commodity prices or implications of interest rates on exchange rates, or the relationship between interest rates and stock exchange prices, etc. Either commodity prices are compared with exchange rates or inflation rates or interest rate movements are compared with exchange rate fluctuations. None of the studies have highlighted the comparison between commodity sub-indices with standard benchmark interest rate index, LIBOR.

The objective of this study to compare commodity indices returns by benchmarking each index against the LIBOR rate. Agriculture, energy and precious metals - three indices have been considered for this analysis. LIBOR as standard interest rate index has been taken for benchmarking. This study can provide insights to commodity producers, manufacturers, investors, and financial institutions by evaluating returns characteristics of commodity indices.

\section{METHODOLOGY}

To determine risk and return characteristics of commodity index vs. LIBOR, three statistical values are calculated namely, Expected Return (ER), Standard Deviation and Geometric Mean (multiplicative mean). The calculated values will help in identifying which investment is better in terms of higher returns with minimum risk. We have used Moving Averages (MA) time series methodology to estimate the future trend of indices. We have followed the steps below to estimate the simple forecasting model of expected return, standard deviation and geometric mean for each index.

1. Consider the dataset of each index

2. Normalize the data

3. Perform exploratory analysis (plot the chart and decompose to see trend, seasonality and error component of each index)

4. Calculate ER, Standard Deviation and Geometric Mean of each index

a. Simple Average

b. Holt-Winters Exponential Smoothing

c. ARIMA

5. Benchmark each commodity index against LIBOR

6. Analyze the results
Before we proceed to perform analysis, let us understand the formula of each metric used in the comparison of each index.

\section{EXPECTED RETURN}

The expected return of an Index is the weighted average of the expected annualized returns. The formula is -

$$
E\left(R_{I}\right)=\sum_{j=1}^{m} W_{j} E\left(R_{j}\right)
$$

$\mathrm{E}\left(\mathrm{R}_{\mathrm{I}}\right)$ is expected return of an Index, $j$ is the number of observations, $\mathrm{W}_{j}$ is the weighted average of daily returns that are $E\left(R_{j}\right)$.

\section{Standard Deviation}

The standard deviation of an Index is an annualized risk of the index in percentage unit. The formula is -

$$
\mathrm{S}_{I}=\sqrt{\frac{1}{N-1} \sum_{i=1}^{N}\left(x_{i}-\bar{x}\right)^{2}}
$$

$\mathrm{S}_{I}$ is the standard deviation of Index, $N$ is the number of observations, $i$ is the value of each observation, $x_{i}$ is $x$ variable values and $x_{i}$ is the sample mean.

\section{Geometric Mean}

The geometric mean is another criterion to calculate return using multiplicity approach. The formula is -

$$
\left(\prod_{i=1}^{N} x_{i}\right)^{\frac{I}{N}}=\sqrt[n]{a_{1}, a_{2}, \ldots . a_{n}}
$$

The equation states the $\mathrm{n}^{\text {th }}$ root of the product of the number of observations. This metric is generally used for estimating future growth rates or interest rates based on historical data.

To gain more clarity in the analysis, we have used another time series forecasting method called Holt-Winters multiplicative method. In this method, we have taken 3 smoothing parameters $\alpha, \beta^{*}$, and $\gamma$. This time series forecasting method is used to calculate the expected trend of returns and risk parameters. The return is shown as a point estimate and error terms (risk) are shown as Mean Absolute Error (MAE), Mean Absolute Percentage Error (MAPE), and Mean Absolute Squared Error (MASE). Autoregressive Integrated Moving Average (ARIMA) is another method that is used to compute the future prices of these indices. This method is used for short-term forecasting of time series data available.

Let us look at the formula for each error term described above.

$$
M A E=\left(\left|e_{i}\right|\right)
$$

Where, $e_{i}$ is forecast error which equals to $y_{i}-\hat{y}_{i} \cdot y_{i}$ is $\mathrm{i}^{\text {th }}$ observation and $\hat{y}_{i}$ is a forecast of $y_{i}$. 


$$
M A P E=\left(\left|p_{i}\right|\right)
$$

Where $p_{i}$ is equals to $100 e_{i / y_{i}}$

$$
\operatorname{MASE}=\left(\left|q_{j}\right|\right)
$$

Where $q_{j}$ is independent of scaled error.

$$
q_{j}=\frac{e_{j}}{\frac{1}{N} \sum_{i=1}^{N}\left|y_{i}-\widehat{y}_{l}\right|}
$$

\section{S\&P GSCI Agriculture Index (SGK) Vs Overnight London} Interbank Offered Rate (LIBOR), based on U.S Dollar

A daily closing price of SGK is considered from 10/9/2001 to $12 / 30 / 2016$. To measure prices against LIBOR prices, the metric is converted to the comparable unit. The log function is used to normalize the prices of SGK and LIBOR. Here, the assumption is that the prices of these 2 indices are distributed log-normally. This would help predict better results in terms of forecasting. The next step is to perform exploratory analysis by decomposing the dataset of SGK and LIBOR.

Agriculture Index (SGK)

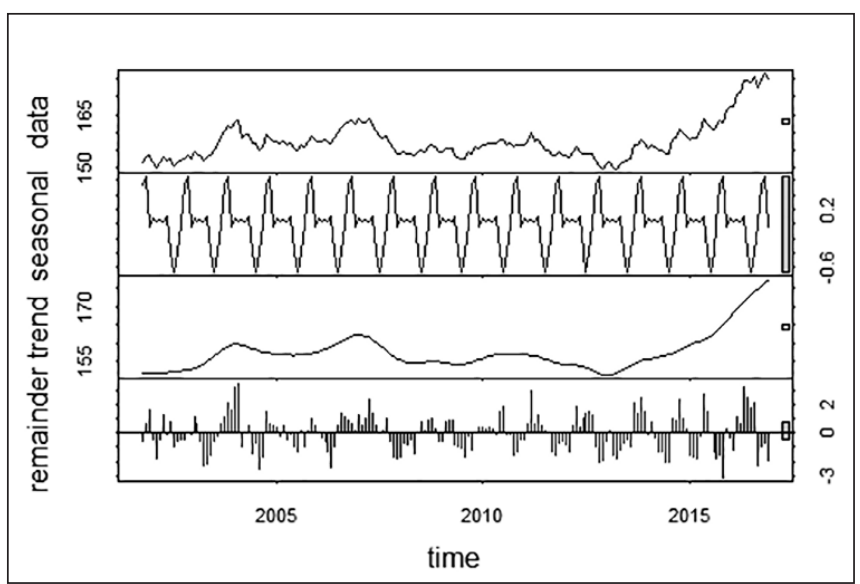

LIBOR

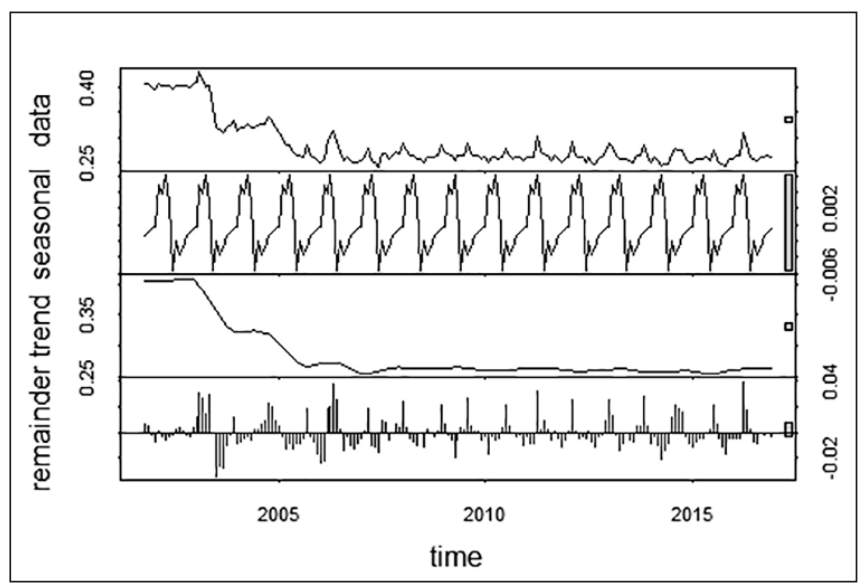

Figure 1: Trend of Agriculture Index (SGK) and LIBOR
Decomposed data clearly indicates an increasing trend for Agriculture Index (SGK) whereas LIBOR indicates a decreasing trend over a period. To analyze further, computation of metrics is used to measure future returns Table 1. shows calculated log values of SGK and LIBOR.

Table 1. Summary Statistics Agriculture Index and LIBOR

\begin{tabular}{|l|l|l|}
\hline Metric & SGK & LIBOR \\
\hline Expected Return & $2 \%$ & $-4 \%$ \\
\hline Standard Deviation (Risk) & $9 \%$ & $36 \%$ \\
\hline Geometric Mean & $1 \%$ & $-10 \%$ \\
\hline Number of Observations & 3727 & 3727 \\
\hline
\end{tabular}

The results indicate that the Agriculture Index (SGK) provides $2 \%$ returns with an annual risk of $9 \%$ whereas LIBOR provides negative returns of $-4 \%$ with an annualized risk of $36 \%$. To get more clarity, we use the multiplicative model to determine return in terms of the geometric mean. SGK provides $1 \%$ annual return whereas LIBOR indicates $-10 \%$ returns. This result also forecasts SGK investment is better than LIBOR return.

\section{Expected Return, Standard Deviation, Geometric Mean (SGK vs. LIBOR) - Recession Period}

We have considered the recession period as one of the macroeconomic variables to observe the risk and return characteristics of SGK and LIBOR. Our extended analysis considering the recession parameter provides support to our findings. Daily time series recession data from 2001 to 2009 is undertaken to compute the expected return, standard deviation and the geometric mean of the two indices. Table 2. shows the calculated risk and return values of SGK and LIBOR.

Table 2. Summary Statistics Agriculture Index and LIBOR (R)

\begin{tabular}{|l|l|l|}
\hline Metric Recession Data & SGK (R) & LIBOR (R) \\
\hline Expected Return & $-1 \%$ & $-80 \%$ \\
\hline Standard Deviation (Risk) & $15 \%$ & $94 \%$ \\
\hline Geometric Mean & $-2 \%$ & $-125 \%$ \\
\hline Number of Observations & 384 & 384 \\
\hline
\end{tabular}

The results indicate that the Agriculture Index (SGK) provides negative $-1 \%$ returns with an annual risk of $15 \%$ whereas LIBOR provides negative returns of $-80 \%$ with an annualized risk of $94 \%$. To get more clarity, we use the multiplicative model to determine returns in terms of the geometric mean. SGK provides $-2 \%$ annual returns whereas LIBOR indicates $-125 \%$ returns. The ratio of risk and return characteristics of these two shows a significant difference.

During the recession period, there were time series data points not showing recession parameter. The analysis 
is conducted on this non-recession period also. We have ascertained the risk and return parameters have shown similar results in comparing risk and return characteristics of commodity indices vs. LIBOR.

Daily time series recession data from 2001 to 2009 for non-recession (NR) period is taken to extend this study. Table

3. shows calculated return values of SGK and LIBOR for the data points that didn't show recession parameters.

Table 3. Summary Statistics Agriculture Index and LIBOR (NR)

\begin{tabular}{|l|l|l|}
\hline Metric Recession Data & SGK (NR) & LIBOR (NR) \\
\hline Expected Return & $22.10 \%$ & $-73 \%$ \\
\hline Standard Deviation (Risk) & $20 \%$ & $104 \%$ \\
\hline Geometric Mean & $20.33 \%$ & $1 \%$ \\
\hline Number of Observations & 413 & 413 \\
\hline
\end{tabular}

The results indicate that the Agriculture Index (SGK) provides $22.10 \%$ return with an annual risk of $20 \%$ whereas LIBOR provides a negative return of $-73 \%$ with an annualized risk of $104 \%$. To get more clarity, we use the multiplicative model to determine return in terms of the geometric mean. SGK provides $20.33 \%$ annual return whereas LIBOR indicates $1 \%$ return. This result also forecasts SGK investment is better than LIBOR returns.

The next section provides another method of forecasting risk and returns in terms of Weighted Average methodology founded by Holt, Winters, and Brown.

\section{Holt-Winters Multiplicative Method (SGK vs. LIBOR)}

Holt-Winters forecasting model is used to see a future trend in terms of expected return and risk. SGK and LIBOR time series data is used as input parameters along with $\mathrm{D}, \mathrm{\square}$ and $\square$ smoothing parameters. The results parameters examined are point forecast for expected return and MAE, MAPE and MASE for risk characteristics of investments.Table 4. shows calculated output parameters for SGK and LIBOR.

Table 4. Holt-Winter Model Summary Statistics (SGK vs. LIBOR)

\begin{tabular}{|l|l|l|}
\hline Metric & SGK & LIBOR \\
\hline Expected Return (Point Estimate) & $2.63 \%$ & $0.25 \%$ \\
\hline MAE (Risk Parameter) & $0.002 \%$ & $0.01 \%$ \\
\hline MAPE (Risk Parameter) & $0.10 \%$ & $3.92 \%$ \\
\hline MASE (Risk Parameter) & $0.31 \%$ & $0.52 \%$ \\
\hline Number of Observations & 3727 & 3727 \\
\hline
\end{tabular}

The results state that SGK return shows a $2.63 \%$ increase with $0.10 \%$ annualized risk and LIBOR returns shows $0.25 \%$ with $3.92 \%$ annualized risk. The forecast result in the plot diagram clearly shows increased return for SGK and LIBOR.

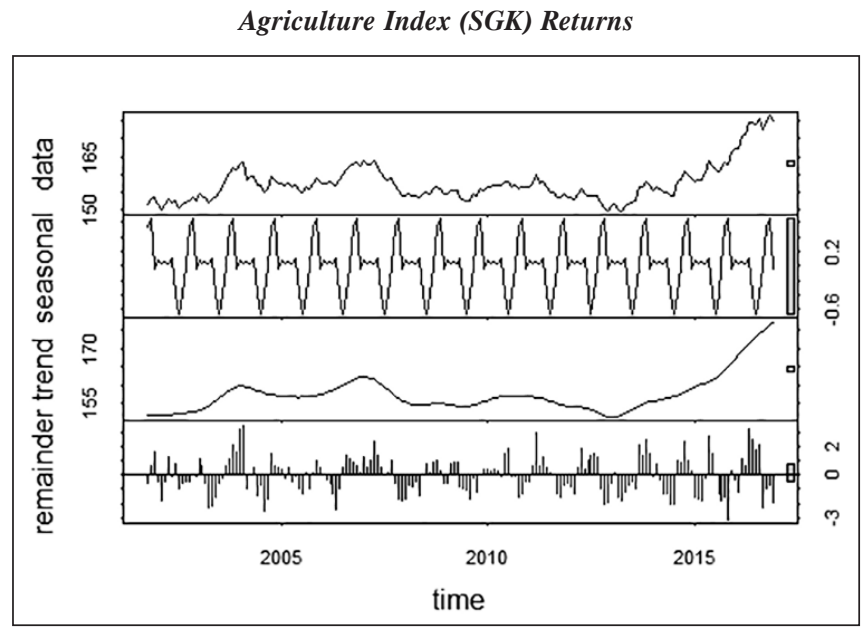

LIBOR Returns

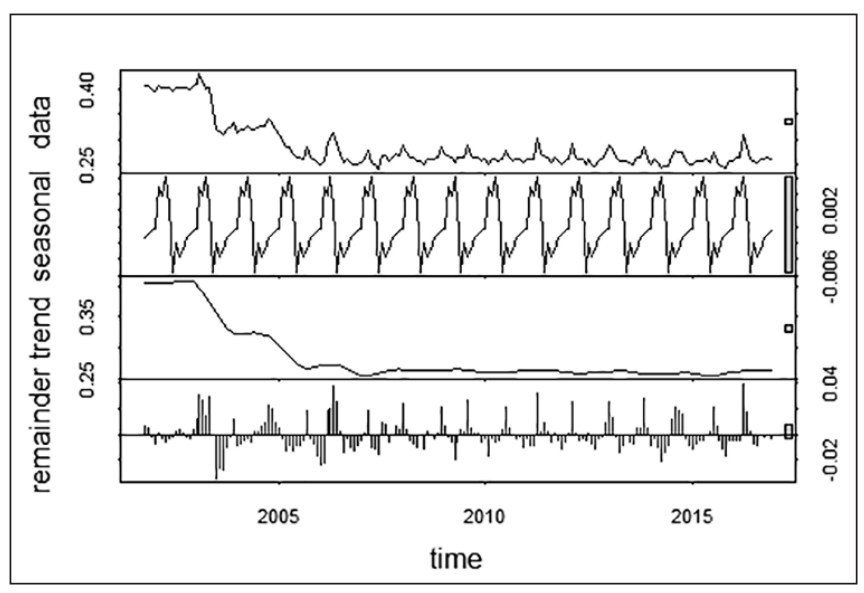

Figure 2: Returns of Agriculture Index (SGK) and LIBOR

Another forecasting method, Autoregressive Integrated Moving Averages (ARIMA) is used to evaluate the returns offered by SGK and LIBOR.

SGK and LIBOR time series data is used as an input with $\mathrm{p}, \mathrm{d}, \mathrm{q}$ as smoothening parameters. ARIMA $(0,1,0)$ is used for SGK and ARIMA $(1,1,1)$ is used for LIBOR. The results parameters examined are point forecast for expected returns and MAE, MAPE and MASE for risk characteristics of investments. Table 5. shows calculated output parameters for SGK and LIBOR.

Table 5. ARIMA Summary Statistics (SGK vs. LIBOR)

\begin{tabular}{|l|l|l|}
\hline Metric & SGK (ARIMA) & LIBOR (ARIMA) \\
\hline Expected Return (Point Estimate) & $2.24 \%$ & $0.25 \%$ \\
\hline MAE (Risk Parameter) & $0.002 \%$ & $0.008 \%$ \\
\hline MAPE (Risk Parameter) & $0.12 \%$ & $3.13 \%$ \\
\hline MASE (Risk Parameter) & $0.26 \%$ & 0.41 \\
\hline Number of Observations & 3727 & 3727 \\
\hline
\end{tabular}


The results state that SGK return shows a $2.24 \%$ increase with $0.12 \%$ annualized risk and LIBOR returns shows $0.25 \%$ with $3.13 \%$ annualized risk. The forecast results are close to the results provided by the Holt-Winters methodology.

\section{S\&P GSCI Energy Index (SGJ) Vs Overnight London Interbank Offered Rate (LIBOR), based on U.S Dollar}

A daily closing price of SGJ is considered from $10 / 9 / 2001$ to $12 / 30 / 2016$. To measure prices against LIBOR prices, the metric is converted to the comparable unit. The log function is used to normalize the prices of SGP and LIBOR. Here, the assumption is that the prices of these 2 indices are distributed log-normally. This would help predict better results in terms of forecasting. The next step is to perform exploratory analysis by decomposing

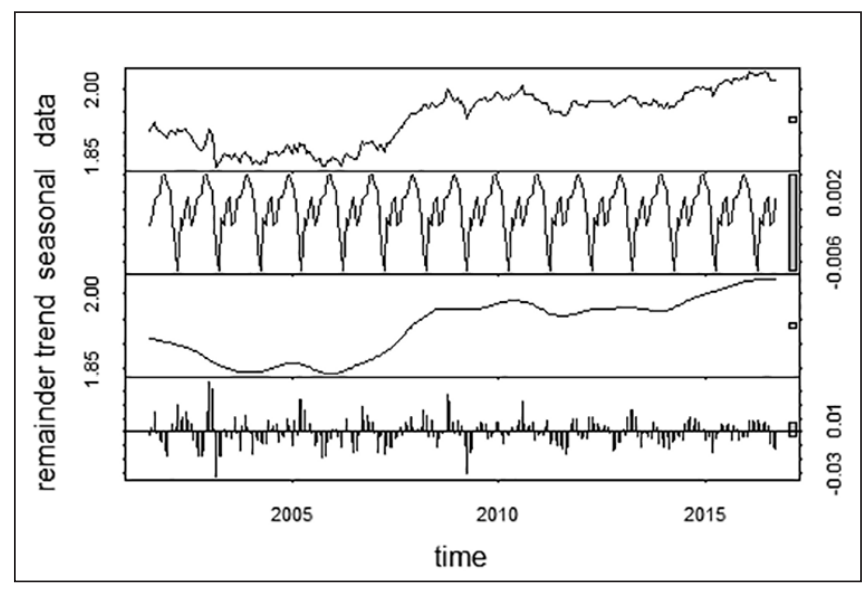

the dataset of SGJ and LIBOR.

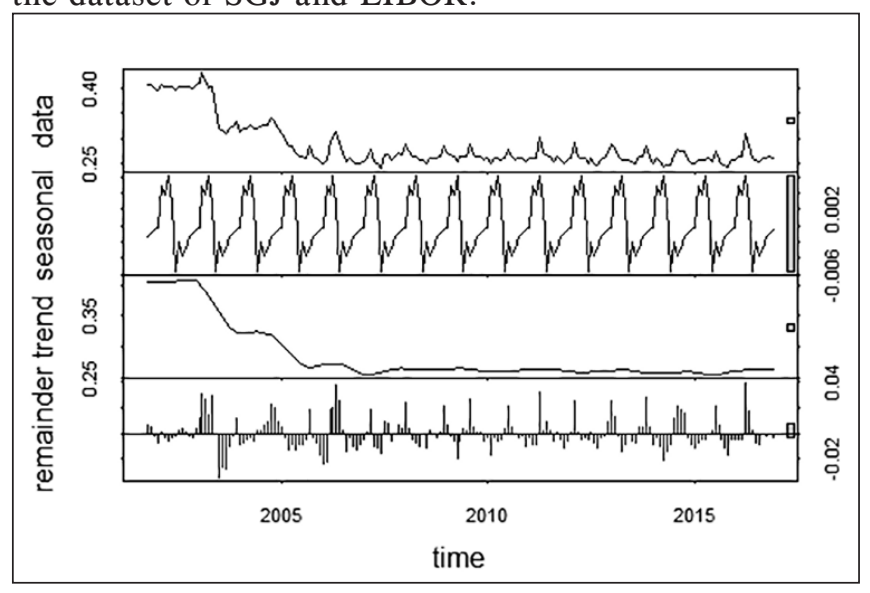

Energy Index (SGJ)

LIBOR

Figure 3: Trend of Energy Index (SGJ) and LIBOR

Decomposed data clearly indicates an increasing trend for Energy Index (SGJ) whereas LIBOR indicates a decreasing trend over a period. To analyze further, computation of metrics is used to measure future returns. Table 6. shows calculated log values of SGJ and LIBOR.
Table 6. Summary Statistics Energy Index and LIBOR

\begin{tabular}{|l|l|l|}
\hline Metric & SGJ & LIBOR \\
\hline Expected Return & $2 \%$ & $-4 \%$ \\
\hline Standard Deviation (Risk) & $14 \%$ & $36 \%$ \\
\hline Geometric Mean & $1 \%$ & $-10 \%$ \\
\hline Number of Observations & 3728 & 3728 \\
\hline
\end{tabular}

The results indicate that Energy Index (SGJ) provides 2\% returns with an annual risk of $14 \%$ whereas LIBOR provides negative returns of $-4 \%$ with an annualized risk of $36 \%$. To get more clarity, we use the multiplicative model to determine returns in terms of the geometric mean. SGJ provides $1 \%$ annual return whereas LIBOR indicates $-10 \%$ returns.

\section{Expected Return, Standard Deviation, Geometric Mean (SGJ vs. LIBOR) - Recession Period}

For the SGJ Index and LIBOR Index, we have extended the analysis considering the recession parameter provides support to our findings. Daily time series recession data from 2001 to 2009 is undertaken to compute the expected return, standard deviation and the geometric mean of the two indices.

Table 7. Summary Statistics Energy Index and LIBOR (R)

\begin{tabular}{|l|l|l|}
\hline Metric Recession Data & SGJ (R) & LIBOR (R) \\
\hline Expected Return & $-10 \%$ & $-80 \%$ \\
\hline Standard Deviation (Risk) & $21 \%$ & $94 \%$ \\
\hline Geometric Mean & $-12 \%$ & $-125 \%$ \\
\hline Number of Observations & 384 & 384 \\
\hline
\end{tabular}

The results indicate that Energy Index (SGJ) provides negative $-10 \%$ returns with an annual risk of $15 \%$ whereas LIBOR provides negative returns of $21 \%$ with an annualized risk of $94 \%$. To get more clarity, we use the multiplicative model to determine returns in terms of the geometric mean. SGJ provides $-12 \%$ annual returns whereas LIBOR indicates $-125 \%$ returns. The ratio of risk and return characteristics of these two shows a significant difference.

We have further computed returns characteristics of these indices during non-recession (NR) period.

Daily time series non-recession data from 2001 to 2009 is undertaken to compute the expected return, standard deviation and the geometric mean of the two indices.

Table 8. Summary Statistics Energy Index and LIBOR (NR)

\begin{tabular}{|l|l|l|}
\hline Metric Recession Data & SGJ (NR) & LIBOR (NR) \\
\hline Expected Return & $33.15 \%$ & $-73 \%$ \\
\hline Standard Deviation (Risk) & $39 \%$ & $104 \%$ \\
\hline Geometric Mean & $26.91 \%$ & $1 \%$ \\
\hline Number of Observations & 412 & 412 \\
\hline
\end{tabular}


The results indicate that Energy Index (SGJ) provides negative $33.15 \%$ returns with an annual risk of $39 \%$ whereas LIBOR provides negative returns of $-73 \%$ with an annualized risk of $104 \%$. To get more clarity, we use the multiplicative model to determine returns in terms of the geometric mean. SGJ provides $26.91 \%$ annual return whereas LIBOR indicates $1 \%$ return.

\section{Holt-Winters Multiplicative Method (SGJ vs. LIBOR)}

SGJ and LIBOR time series data is used as input parameters along with $\alpha, \beta *$ and $\gamma$ smoothing parameters. The results parameters examined are point forecast for expected returns and MAE, MAPE and MASE for risk characteristics of investments Table 9. shows calculated output parameters for SGJ and LIBOR.

Table 9. Holt-Winter Model Summary Statistics (SGJ vs. LIBOR)

\begin{tabular}{|l|l|l|}
\hline Metric & SGJ & LIBOR \\
\hline Expected Return (Point Estimate) & $2.29 \%$ & $0.25 \%$ \\
\hline MAE (Risk Parameter) & $0.004 \%$ & $0.01 \%$ \\
\hline MAPE (Risk Parameter) & $0.19 \%$ & $3.92 \%$ \\
\hline MASE (Risk Parameter) & $0.28 \%$ & $0.52 \%$ \\
\hline Number of Observations & 3728 & 3728 \\
\hline
\end{tabular}

The results state that SGJ return shows a $2.29 \%$ increase with $0.19 \%$ annualized risk and LIBOR return shows $0.25 \%$ with $3.92 \%$ annualized risk. The forecast result in the plot diagram clearly shows increased return for SGJ and LIBOR.

Energy Index (SGJ) Returns

\section{Forecasts from Holt-Winters' multiplicative method}

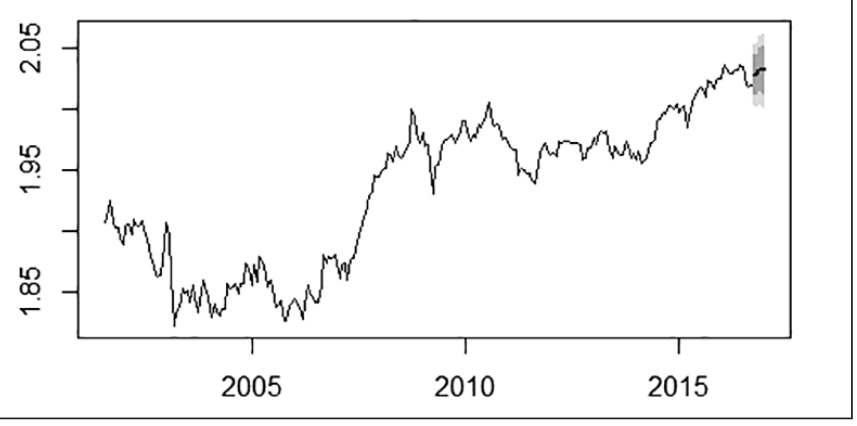

LIBOR Returns

\section{Forecasts from Holt-Winters' multiplicative method}

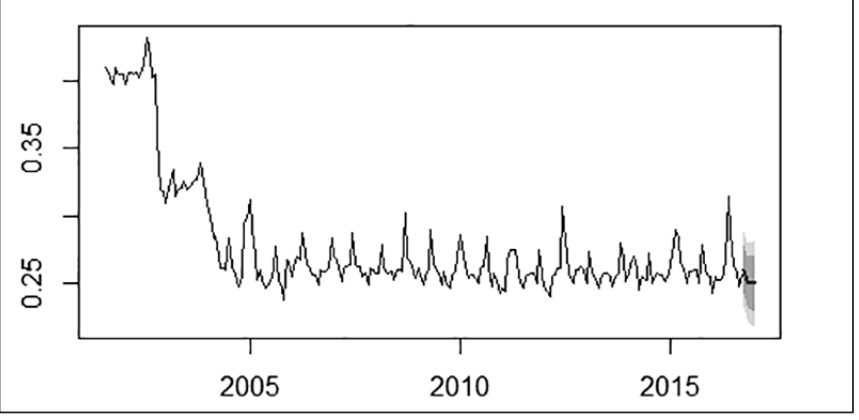

Figure 4: Returns of Energy Index (SGJ) and LIBOR
Autoregressive Integrated Moving Averages (ARIMA) is also used to evaluate the returns offered by SGJ and LIBOR.

SGJ and LIBOR time series data is used as an input with $\mathrm{p}, \mathrm{d}, \mathrm{q}$ as smoothening parameters. ARIMA $(0,1,0)$ is used for SGK and ARIMA $(1,1,1)$ is used for LIBOR. The results parameters examined are point forecast for expected returns and MAE, MAPE and MASE for risk characteristics of investments.Table 10. shows calculated output parameters for SGJ and LIBOR.

Table 10. ARIMA Summary Statistics (SGJ vs. LIBOR)

\begin{tabular}{|l|l|l|}
\hline Metric & SGJ (ARIMA) & LIBOR (ARIMA) \\
\hline $\begin{array}{l}\text { Expected Return (Point } \\
\text { Estimate) }\end{array}$ & $2.62 \%$ & $0.25 \%$ \\
\hline MAE (Risk Parameter) & $0.01 \%$ & $0.008 \%$ \\
\hline MAPE (Risk Parameter) & $0.43 \%$ & $3.13 \%$ \\
\hline MASE (Risk Parameter) & $0.16 \%$ & $0.41 \%$ \\
\hline Number of Observations & 3727 & 3727 \\
\hline
\end{tabular}

The results state that SGK return show $2.62 \%$ increase with $0.01 \%$ annualized risk and LIBOR returns shows $0.25 \%$ with $3.13 \%$ annualized risk. The forecast results are close to the results provided by Holt-Winters methodology.

\section{S\&P GSCI Precious Metals Index Vs Overnight London} Interbank Offered Rate (LIBOR), based on U.S Dollar

A daily closing price of SGP is considered from $10 / 9 / 2001$ to $12 / 30 / 2016$. To measure prices against LIBOR prices, the metric is converted to the comparable unit. The log function is used to normalize the prices of SGP and LIBOR. Here, the assumption is that the prices of these 2 indices are distributed log-normally. The next step is to perform exploratory analysis by decomposing the dataset of SGP and LIBOR.

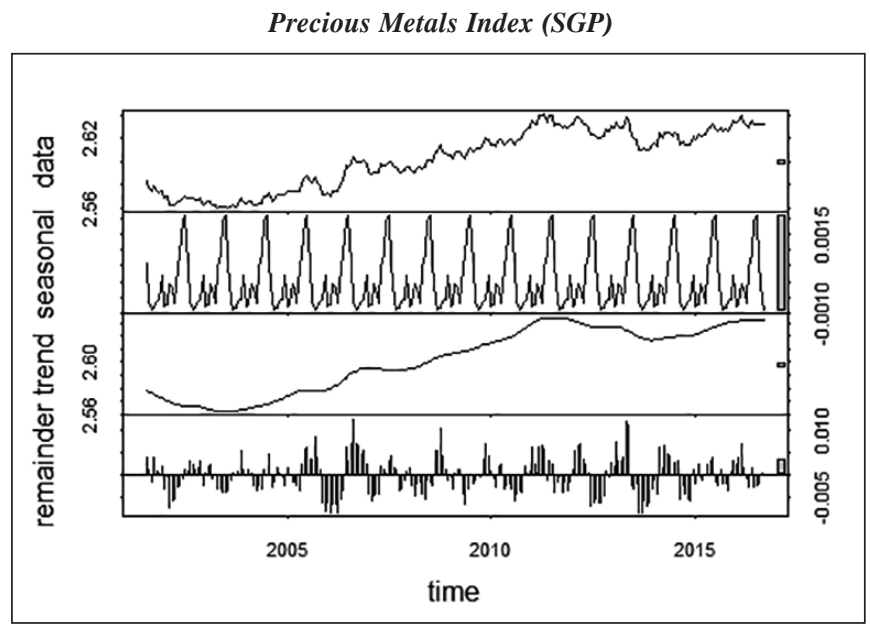


LIBOR

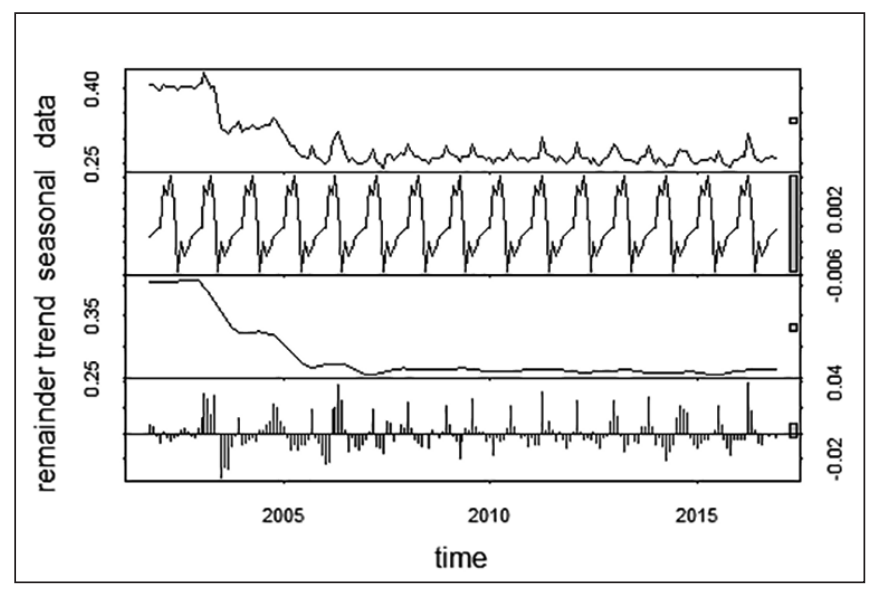

Figure 5: Trend of Precious Metals Index (SGP) and LIBOR

Decomposed data clearly indicates an increasing trend for Precious Metals Index (SGP) whereas LIBOR indicates a decreasing trend over a period. To analyze further, computation of metrics is used to measure future returns. Table 11. shows calculated log values of SGP and LIBOR.

Table 11. Summary Statistics Precious Metals Index and LIBOR

\begin{tabular}{|l|l|l|}
\hline Metric & SGP & LIBOR \\
\hline Expected Return & $4 \%$ & $-4 \%$ \\
\hline Standard Deviation (Risk) & $9 \%$ & $36 \%$ \\
\hline Geometric Mean & $4 \%$ & $-10 \%$ \\
\hline Number of Observations & 3727 & 3727 \\
\hline
\end{tabular}

The results indicate that the Precious Metals Index (SGP) provides $4 \%$ return with an annual risk of $9 \%$ whereas LIBOR provides negative return of $-4 \%$ with an annualized risk of $36 \%$. To get more clarity, we use the multiplicative model to determine return in terms of the geometric mean. SGP provides $4 \%$ annual return whereas LIBOR indicates $-10 \%$ return.

\section{Expected Return, Standard Deviation, Geometric Mean (SGP vs. LIBOR) - Recession Period}

For SGP Index and LIBOR Index, we have extended the analysis considering the recession parameter provides support to our findings. Daily time series recession data from 2001 to 2009 is undertaken to compute the expected return, standard deviation and the geometric mean of the two indices.

\begin{tabular}{|l|l|l|}
\hline Metric Recession Data & SGP (R) & LIBOR (R) \\
\hline Expected Return & $5 \%$ & $-80 \%$ \\
\hline Standard Deviation (Risk) & $13 \%$ & $94 \%$ \\
\hline Geometric Mean & $4 \%$ & $-125 \%$ \\
\hline Number of Observations & 384 & 384 \\
\hline
\end{tabular}

Table 12. Summary Statistics Precious Metals Index and LIBOR (R)
The results indicate that the Precious Metals Index (SGP) provides $5 \%$ returns with an annual risk of $13 \%$ whereas LIBOR provides negative returns of $-80 \%$ with an annualized risk of $94 \%$. To get more clarity, we use the multiplicative model to determine returns in terms of the geometric mean. SGP provides $4 \%$ annual return whereas LIBOR indicates $-125 \%$ returns. The ratio of risk and return characteristics of these two shows a significant difference.

We have further computed returns characteristics of these indices during non-recession (NR) period. Daily time series non-recession data from 2001 to 2009 is undertaken to compute the expected return, standard deviation and the geometric mean of the two indices.

Table 13. Summary Statistics Precious Metals Index and LIBOR (NR)

\begin{tabular}{|l|l|l|}
\hline Metric Recession Data & SGP (NR) & LIBOR (NR) \\
\hline Expected Return & $36.46 \%$ & $-73 \%$ \\
\hline Standard Deviation (Risk) & $30 \%$ & $104 \%$ \\
\hline Geometric Mean & $32.70 \%$ & $1 \%$ \\
\hline Number of Observations & 412 & 412 \\
\hline
\end{tabular}

The results indicate that Precious Metals Index (SGP) provides negative $36.46 \%$ returns with an annual risk of $30 \%$ whereas LIBOR provides negative returns of $-73 \%$ with an annualized risk of $104 \%$. To get more clarity, we use the multiplicative model to determine returns in terms of the geometric mean. SGP provides $32.70 \%$ annual return whereas LIBOR indicates $1 \%$ returns.

\section{Holt-Winters Multiplicative Method (SGP vs. LIBOR)}

SGP and LIBOR time series data is used as input parameters along with $\square, \square$ and $\square$ smoothing parameters. The results parameters examined are point forecast for expected returns and MAE, MAPE and MASE for risk characteristics of investments. Table 14. shows calculated output parameters for SGP and LIBOR.

Table 14. Holt-Winter Model Summary Statistics (SGP vs. LIBOR)

\begin{tabular}{|l|l|l|}
\hline Metric & SGP & LIBOR \\
\hline Expected Return (Point Estimate) & $2.63 \%$ & $0.25 \%$ \\
\hline MAE (Risk Parameter) & $0.002 \%$ & $0.01 \%$ \\
\hline MAPE (Risk Parameter) & $0.10 \%$ & $3.92 \%$ \\
\hline MASE (Risk Parameter) & $0.31 \%$ & $0.52 \%$ \\
\hline Number of Observations & 3727 & 3727 \\
\hline
\end{tabular}

The results state that SGP return shows a $2.63 \%$ increase with $0.10 \%$ annualized risk and LIBOR returns shows $0.25 \%$ with $3.92 \%$ annualized risk. The forecast result in the plot diagram clearly shows increased returns for SGP and LIBOR. 
Precious Metals Index (SGP) Returns

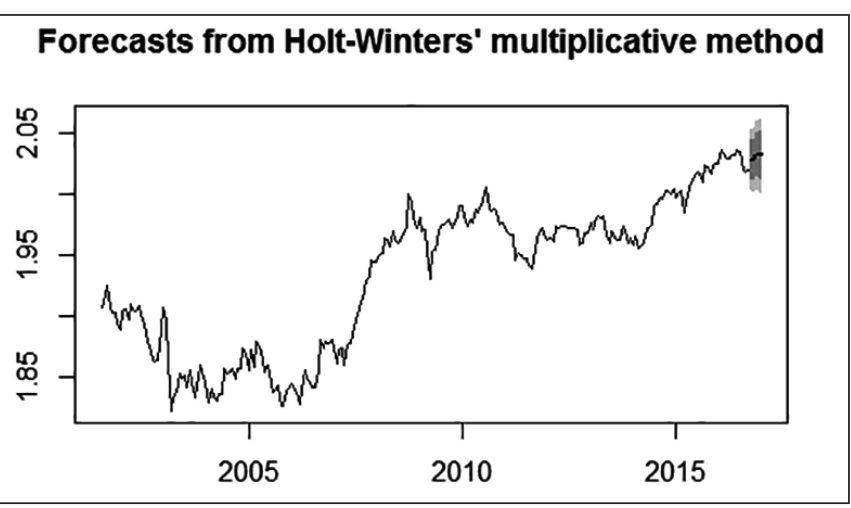

LIBOR Returns

Forecasts from Holt-Winters' multiplicative method

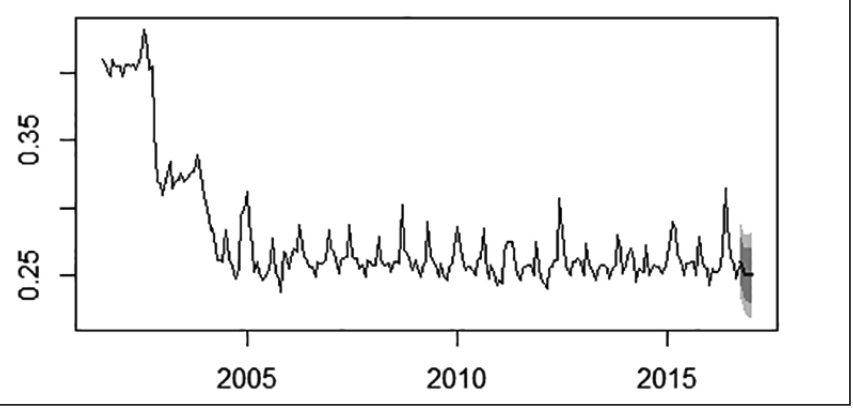

Figure 6: Returns of Precious Metals Index (SGP) and LIBOR

Autoregressive Integrated Moving Averages (ARIMA) is also used to evaluate the returns offered by SGP and LIBOR.

SGP and LIBOR time series data is used as an input with p, d, q as smoothening parameters. ARIMA $(0,1,0)$ is used for SGP and ARIMA $(1,1,1)$ is used for LIBOR. The results parameters examined are point forecast for expected returns and MAE, MAPE and MASE for risk characteristics of investments. Table 15. shows calculated output parameters for SGP and LIBOR.

Table 15. ARIMA Summary Statistics (SGP vs. LIBOR)

\begin{tabular}{|l|l|l|}
\hline Metric & SGP (ARIMA) & LIBOR (ARIMA) \\
\hline Expected Return (Point Estimate) & $2.63 \%$ & $0.25 \%$ \\
\hline MAE (Risk Parameter) & $0.002 \%$ & $0.008 \%$ \\
\hline MAPE (Risk Parameter) & $0.09 \%$ & $3.13 \%$ \\
\hline MASE (Risk Parameter) & $0.30 \%$ & $0.41 \%$ \\
\hline Number of Observations & 3727 & 3727 \\
\hline
\end{tabular}

The results state that SGP return shows a $2.63 \%$ increase with $0.002 \%$ annualized risk and LIBOR returns shows $0.25 \%$ with $3.13 \%$ annualized risk. The forecast results are close to the results provided by the Holt-Winters methodology.

\section{RESULT ANALYSIS}

From the above analysis and forecasting, an annualized return of all the above commodity indices offers higher expected returns compared to LIBOR expected returns. All the three commodity indices are the best bet compared to the annualized LIBOR return of $-4 \%$ and $36 \%$ annual risk.

Analysis using Recession parameters also have shown similar results in forecasting returns of commodity indices and LIBOR. The results indicated that the annualized return of commodity indices is higher than LIBOR return of $-80 \%$ with $94 \%$ annual risk.

Holt-Winter Multiplicative forecasting method also has shown the similar results of the annualized return of commodity indices being higher than LIBOR returns. Looking at risk and return characteristics of commodity indices, hedgers and speculators can consider commodity index investment in their portfolio. LIBOR, on the other hand, shows small returns with higher risk compared to commodity indices.

\section{CONCLUSIONS}

Most of the previous studies analyzed the correlation between LIBOR and treasury rates, determining linkages between commodity markets and stock markets, or investigating the relationship of macroeconomic variables with asset classes. No study has compared risk and return parameters of commodity indices vs. LIBOR. We have conducted comparison studies with a focus on commodity futures indices and LIBOR.

In this study, we analyzed risk and return characteristics of indices of two asset classes, commodities, and interest rates. The analysis result indicates that the commodity index investment provides better return compared to LIBOR return. LIBOR has shown higher risk and low returns compared to commodity indices.

LIBOR interest rates data taken for our analysis may have a potential bias connected with the manipulation of interbank lending rates by various financial institutions, known as the LIBOR scandal.

Our study witnessed that there is risk associated with both, commodity indices and LIBOR. Comparison study highlights the risk of commodity index is smaller compared to its return whereas LIBOR shows higher risk compared to its return in recession, non-recession and overall periods. Further research can be carried out by analyzing the risk and return characteristics of various single commodity indices like carbon index or lead index against LIBOR or other interest rate benchmarks.

\section{REFERENCES}

Akram F. Q., (2009): Commodity prices, interest rates and the dollar, Energy Economics 2009; 31(6): 838-851.

Braml H., (2016): The manipulation of LIBOR and related interest rates, Studies in Economics and Finance 2016; 33(1): 106125. 
Covrig V., Low B. S., Melvin M., (2004): A Yen is not a Yen: TIBOR/LIBOR and the determinants of the 'Japan Premium', Journal of Financial and Quantitative Analysis 2004; 39: 193208.

Foerster S.R., Sapp S.G., (2003): How do Interest Rate Changes affect Equities?, Canadian Investment Review Spring 2003; 16(1): 26-34.

Frankel JA. (2012): Commodity prices and interest rates, Outlook Economic Data and Commentary 2012; 9(10):1-10.

Gruber J.W., Vigfusson R.J., (2018): Interest Rates and the Volatility and Correlation of Commodity Prices, Macroeconomic Dynamics, Cambridge University Press 2018; 22(3): 600-619.

Günay S., (2015): Stock Market Liquidity and O/N LIBOR Rates: A Study for PIGS Countries and Turkey, Mediterranean Journal of Social Sciences 2015; 6(3): 297-305.

Kohlscheen E., Avalos F.H., Schrimpf A., (2017): When the Walk is not Random: Commodity Prices and Exchange Rates, International Journal of Central Banking 2017; 13(2): 121-157.

Moss J. D., Moss G. J., (2010): Variables Explaining Bank Stock Prices, The Journal of Applied Business Research 2010; 26(4): 9-12.

Nordin N., Nordin S., \& Ismail R., (2014): The Impact of Commodity Prices, Interest Rate and Exchange Rate on Stock Market Performance: An Empirical Analysis from Malaysia, Malaysian Management Journal 2014; 18: 39-52.

Reicher C.P., Utlaut J. F., (2010): The relationship between oil prices and long-term interest rates, Kiel Institute for the World Economy, Kiel Working Paper No. 16372010.

Sari R., Soytas U., (2006): The relationship between stock returns, crude oil prices, interest rates, and output: evidence from a developing economy, The Empirical Economics Letters 2006; 5(4).

Scherr B. A., Madsen H. C., (1983): Observations on the relationship between agricultural commodity prices and real interest rates, The Journal of Futures Markets 1983; 3(1): 47-54.

Schnabel J.A., (2010): Interest rates, commodity prices, and the cost $\square$ of $\bigsqcup$ carry model, The Journal of Risk Finance 2010; 11(2): 221-223.

Tafa J.,(2015): Relationship between Exchange Rates and Interest Rates: Case of Albania, Mediterranean, Journal of Social Sciences 2015; 6(4): 163-170. 
